\title{
BMJ Open Enhancing the learning and transfer of preprocedural communication skills during clerkship using audio-visual material: a prospective case-controlled study over 2 years
}

\author{
Dung-Hung Chiang, ${ }^{1,2,3}$ Chung-Ting Chen, ${ }^{2,3,4}$ Tse-Yao Wang, ${ }^{2,3,4}$ \\ Ying-Ying Yang (1) , 2,3,5,6 Chia-Chang Huang, ${ }^{2,3,5,7}$ Tzu-Hao Li, ${ }^{6,8}$ \\ Shiau-Shian Huang, ${ }^{3,5,9}$ Shou-Yen Kao, ${ }^{9}$ Chen-Huan Chen, ${ }^{2,3,5,9}$ \\ Ming-Chih Hou, ${ }^{3,8,10}$ Wayne Huey-Herng Sheu ${ }^{2,3,8,11}$
}

To cite: Chiang D-H, Chen C-T, Wang T-Y, et al. Enhancing the learning and transfer of preprocedural communication skills during clerkship using audio-visual material: a prospective case-controlled study over 2 years. BMJ Open 2022;12:e055953. doi:10.1136/ bmjopen-2021-055953

- Prepublication history for this paper is available online. To view these files, please visit the journal online (http://dx.doi. org/10.1136/bmjopen-2021 055953).

D-HC and C-TC are joint first authors.

Received 01 August 2021 Accepted 16 December 2021

Check for updates

(C) Author(s) (or their employer(s)) 2022. Re-use permitted under CC BY-NC. No commercial re-use. See rights and permissions. Published by BMJ.

For numbered affiliations see end of article.

Correspondence to

Dr Ying-Ying Yang;

yangyy@vghtpe.gov.tw

\section{ABSTRACT}

Objective/design/setting This study aims to develop preprocedural communication-specific framework that emphasises the use of audiovisual materials and compares its acceptability by trainees with a regular module.

Trainees Between October 2018 and July 2021, 96 medical clerks were enrolled and randomly divided into regular and intervention groups. Another 48 trainees whose did not join the framework-based training but complete self-assessments were enrolled as the control group.

Interventions In the intervention training module, the key steps of preprocedural communication-specific skills were structuralised into a framework using the acronym of OSCAR.

Primary and secondary outcome measures This study compared the acceptability of trainees for two modules by measuring the degree of increase in the end-of-rotation and follow up (4 weeks later) competency from baseline by trainees' self-assessments and physician assessments after serial trainings.

Results In comparison with regular group trainees, greater degree of improvements (framework- 1 statement: $111 \% \pm 13 \%$ vs $27 \% \pm 5 \%, p<0.001$; framework- 2 statement: $77 \% \pm 9 \%$ vs $48 \% \pm 2 \%, p<0.05$; skill- 1 statement: $105 \% \pm 9 \%$ vs $48 \% \pm 3 \%, p<0.001$ ); skill-2 statement: $71 \% \pm 11 \%$ vs $50 \% \pm 9 \%, p<0.05$ ) were noted in the framework-related and skill-related statement 1-2 (the familiarity and confidence to use the framework and skills) than those of intervention group. At the end-of-rotation stage, the trainees ability to use the 'A-step: using audiovisual materials' of the OSCAR was significantly improved $(229 \% \pm 13 \%, p<0.001)$, compared with other steps. In the intervention group, the degree of improvement of the end-of-rotation data of trainees' selfassessment from baseline was significantly correlated with the degree of the improvement in physicians' assessment data in the aspects of skills, framework and steps in framework $(R=0.872, p<0.01 ; R=0.813, p<0.001$; $R=0.914, p<0.001)$.
Strengths and limitations of this study

- Through serial baseline, end-of-rotation and followup assessments by trainees and physicians, this study evaluates the acceptability of an intervention module for training preprocedural communication skills of medical clerks during clinical rotation.

- In addition to the regular and intervention groups trainees whose receive training for using a preprocedural communication framework and materials, control group whose did not join regular or intervention group were included for comparison.

- This study was limited to a single site with relative sample size.

- The competency of medical clerks' skills in providing preprocedural communication was observed by physicians in class rather than in a clinical ward.

- The study lacks an evaluation of the degree of transfer of acquired skills by medical clerks in practice via direct work-place assessments and patients' satisfaction.

Conclusions The OSCAR framework-based intervention module is well accepted by medical clerks and motivates them to integrate the acquired skills in clinical practice, which leads to trainees' primary care patients being satisfied with their preprocedural communication.

\section{INTRODUCTION}

Before medical procedures, anxious patients are not easy to satisfy with preprocedural communication if they lack specific audiovisual materials and frameworks. ${ }^{1}$ Effective reduction of preprocedural anxiety depends on doctors' effective and accurate communication skills. ${ }^{2}$ When facing anxious patients, detailed communication can decrease their anxiety, improve periprocedural tolerability 
and increase satisfaction with the medical procedure and care. $^{3-5}$

The Accreditation Council for Graduate Medical Education and the American Board of Medical Specialties have jointly identified communication skills as one of the six core competencies for physicians. ${ }^{6-8}$ Moreover, the Institute of Medicine also specifies communication skills as one of the six essential competencies for effective patient care. The Mini-Clinical Evaluation Exercise (Mini-CEX) and Direct Observation of Procedural Skills (DOPS) are formative assessments of the clinical competency of medical trainees. ${ }^{89}$ The importance of the teaching and assessment of communication skills has been reinforced by the fact that it has been integrated as an importance domain in the mini-CEX and DOPS. ${ }^{10}$

During the ward round, physicians sometimes lack sufficient time to provide detailed information about medical procedures to patients. Medical clerks, the most junior physicians, are thus the first-line healthcare personnel responsible for communicating with anxious patients about details of the preparation, periprocedural cooperation, postprocedural precaution, etc. The involvement of medical clerks in communication has been reported to enhance their competency and patients' satisfaction. However, recent studies have reported that in complex clinical environments, without adequate framework, structural training and audiovisual materials, medical clerks and patients will fail to engage in effective preprocedural communication. ${ }^{11} 12$ Therefore, it is important to develop an easy-to-remember framework that integrates audiovisual materials for training and assessing the preprocedural communication skills of medical clerks.

For years in our institution, medical clerks were regularly trained and assessed with a regular framework for medical communication, which included the five steps that shown in table $1 .{ }^{13}$ The ACGME has included this regular framework in its toolbox of assessment methods for communication skills. ${ }^{14}$ However, the regular framework did not include essential elements of preprocedural communication, which are difficult to memorise and use in training and assessment of the process (or coherence) of medical communication in a complex clinical environment. ${ }^{15}$ Coherence is important in this context, as it refers to one's understanding of the procedure.

Recent data derived from the use of the mini-CEX and DOPS showed that trainees' competency of preprocedural communication skills was inferior to other domains. ${ }^{10}$ Meanwhile, trainees reported that they have difficulties in transferring the in-class acquired regular framework for preprocedure communication skills to real-world setting due to the complicated clinical environment and the anxiousness of patients. ${ }^{13} 15$ Thus, beginning in October 2018, for easy memorisation and application, the educational committee developed the

Table 1 The content of each step of regular and OSCAR framework for preprocedural communication skills and the trainings for regular, intervention and control group medical clerks in this study

Details of each step in the regular Step-1:Setting the stage framework
Details of each step in the OSCAR framework
O-step: Opening with what, when, why and how

S-step: explaining Subject to be communicated

C-step: Check for patients' understanding of the subjects

A-step: Aid patient
understanding with audiovisual
material

R-step: Recheck patient understanding

\begin{tabular}{|c|c|c|c|}
\hline $\begin{array}{l}\text { Details trainings and assessment } \\
\text { among groups }\end{array}$ & Regular group & Intervention group & Control group \\
\hline 2-hour introductory class & $\sqrt{ }$ & & \\
\hline $\begin{array}{l}\text { Training for using of five steps } \\
\text { OSCAR framework }\end{array}$ & & $\sqrt{ }$ & \\
\hline $\begin{array}{l}\text { Complete baseline, end- } \\
\text { of-rotation, follow-up self- } \\
\text { assessments }\end{array}$ & $\sqrt{ }$ & $\sqrt{ }$ & $\sqrt{ }$ \\
\hline $\begin{array}{l}\text { Receive baseline and end- } \\
\text { of-rotation assessments from } \\
\text { physicians }\end{array}$ & $\sqrt{ }$ & $\sqrt{ }$ & \\
\hline
\end{tabular}


OSCAR, which includes the 5 steps of Opening, clarifying the Subject to be discussed, Checking patients' understanding of the subject, Aiding patient understanding with procedure-specific audiovisual (audiovisual) materials, and Rechecking the patients' understanding of the subject based on the regular communication framework. The OSCAR framework that shown in table 1 emphasises audiovisual and acronym methods for training on preprocedural communication. ${ }^{131516}$

It is important to build trainees' ability to effectively communicate the procedure and risk of interventions to patients. In particular, involvement of medical clerks in communication can enhance their competency and patients' satisfaction. ${ }^{17}$ It had been reported that anxious patients are prone to feeling dissatisfaction in regard to receive information simply through verbal communication. ${ }^{18}$ To enhance preprocedural satisfaction and maximise information gain, recent studies have suggested the use of audiovisual materials in) the 'A step: Aid patient understanding' step of the OSCAR communication framework. ${ }^{18-21}$

Taken together, this study aims to evaluate the acceptability of the implementation of the audiovisual materialsintegrated preprocedural communication-specific OSCAR framework in teaching and assessing the preprocedural communication skills of medical clerks during clinical rotation. At the end-of-rotation and follow-up stages, the degree of improvement and retention effects were compared between groups. Finally, the correlation between the degree of improvement from baseline of the trainee self-assessments and physician assessments was evaluated.

\section{METHODS}

\section{Research design and training hypothesis}

In 2016-2017, the annual clerkship surveys revealed that the regular framework-based communication training module did not give medical trainees confidence in providing preprocedural communication. Facing this challenge, the educational committee developed medical procedure-specific audiovisual materials and integrated them into a preprocedural communication-specific framework.

This was a prospective case-controlled study. The training hypotheses for the regular and intervention modules were the same, including experiential learning, structured skill-based learning and learner self-reflection fostering. ${ }^{22} 23$ In both the regular and intervention modules, didactic lecture and small-group role play plus discussion were included in the demonstration, discussion, reflection, practice and feedback phases for training with either the regular or OSCAR framework. ${ }^{11} 1622-24 \mathrm{We}$ proposed that, in comparison with the regular framework, the OSCAR framework, which includes the detailed essential steps of preprocedural communication will enhance the acceptability of training. In particular, the use of audiovisual materials has been emphasised in the OSCAR framework. In this study, we hypothesised that use of the OSCAR framework would lead to better communication with patients than the regular approach, and better than communication seen in the control group.

\section{Participants}

Between August 2019 and July 2021, 48 medical clerks in each group (eight consecutive batches of six students) were enrolled and randomly divided into regular and intervention groups for training of preprocedural communication skills related to central venous catheterisation, bronchoscopy, thoracentesis, lumbar puncture and tracheostomy with either regular or intervention modules in their clinical rotation. In the intervention module, the OSCAR framework was designed for preprocedural communication in-class and in clinical practice. Before implementation in routine training, the OSCAR framework was piloted by testing it with voluntary medical clerks to ensure that the element of preprocedural communication had been integrated appropriately. Additionally, the third group of medical clerks $(n=48)$ whose did not join either regular or intervention groups to receive additional training using a preprocedural communication framework and materials were served as the control group.

\section{Procedure-content of regular five-step and OSCAR-based communication training modules}

In both the regular and OSCAR framework-based modules for preprocedural communication, the same introductory class and end-of-rotation class were arranged and led by certified physicians (table 1). The 2-hour introductory classes began with introductory demonstrations using either the regular or OSCAR framework (30 min), and then featured, interactive discussion regarding using either framework to increase patients' understanding of the details of medical procedures $(30 \mathrm{~min})$, reflection on trainees' real-world experience with procedural communication ( $15 \mathrm{~min})$, dyad practising the regular or OSCAR framework with one standardised patient (SP) (30 min), ultimately concluding with a grouped feedback ( $15 \mathrm{~min}$; figure 1). In contrast to the regular framework-based module, the intervention module emphasised the integration of procedure-specific audiovisual (two-dimensional, three-dimensional, virtual reality) (materials in) class and in clinical practice. Overall, both modules asked trainees to integrate acquired knowledge and skills in communicating with their patients between the introductory and end-of-rotation classes. On the end-of-rotation class, the physician discussed each trainee's real-world experience with practice, provided feedback, demonstrated changeable behaviours through role playing, and ended with group reflection (table 1$).{ }^{22-24}$

\section{Instrumentation for training and assessments}

To increase the efficiency of clinical practice and in-class training, and to achieve a paperless approach, the detailed contents of the training, regular or OSCAR framework, 
Regular or intervention group trainees received framework-based trainings with regular module or intervention module for enhancing pre-procedural communication skills

Introductory class

-All trainees complete the baseline self-assessment

-Physicians trained trainees for giving pre-procedural communication using regular or OSCAR

framework through role-playing demo on standardized patients (SPs)

-Trainees took turn to practice the procedural communication skills of either central venous

catheterization, lumbar puncture, bronchoscopy, thoracentesis, or tracheostomy with SPs.

-Physicians complete the baseline assessment of each trainee' competency control group did not receive framework-based training for pre-procedural communication skills

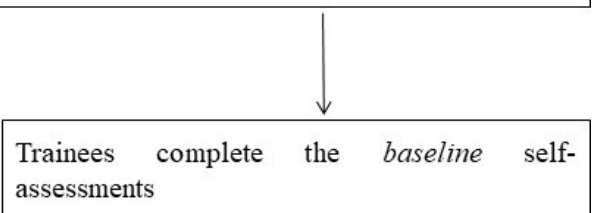

\section{End-of-rotation class}

-Physicians re-demonstrate the skills, framework and steps of frameworks to

enhance trainees' competency of pre-procedural communication.

-Physicians assess trainees' end-of-rotation competency.

-Trainees re-assess their end-of-rotation competency
Trainees are encouraged to integrate their acquired skills in communication with their patients

-

Follow-up at 4 weeks after end-of-rotation

-trainees repeat the self-assessment of their competency for giving preprocedural communication to their patients

-whether apply the acquired skills in primary care and whether patients are satisfied with their skills of preprocedural communication

Figure 1 Schematic flow for additional training of the preprocedural communication skills of medical clerks for the regular framework-based regular module and OSCAR framework-based intervention module. The control group is the third group trainees whose did not belong to either the regular or intervention group.

audiovisual materials and assessment form were packaged in various $\mathrm{QR}$ codes. Users can access the information using various devices, such as smartphones, iPads and laptops. Medical clerks and physicians in the regular, intervention and control groups were asked to complete the baseline and end-of-rotation assessments in table 2 . Additionally, 4 weeks after end-of-rotation, trainees were emailed for follow-up regarding their competency, whether they applied the learnt skills in clinical practice, and patient satisfaction in relation to their preprocedural communication skills.

\section{Patient and public involvement \\ No patient involved}

\section{Statistical analysis}

Data were expressed as mean \pm SD. A significance level of $p=0.05$ was chosen. The significance of difference of baseline and end-of-rotation data of individual groups were compared with paired students $t$ test. Both for the trainees-self-assessment and physicians-assessment, the degree of improvement (per cent changes) of end-ofrotation data from baseline data of the step-1, step-2, step-3, step-4, step-5 in the regular framework of within the regular group were compared with one-way Analysis of Variance (ANOVA). Similarly, the degree of improvement (per cent changes) of end-of-rotation data from baseline data of the O-step, S-step, C-step, A-step and R-step in OSCAR framework within the intervention group were compared with one-way ANOVA with post hoc Tukey
Test. The comparison of the degree of improvement of the skill-related or framework-related competency between regular and intervention group were analysed with Student's t-test. Pearson correlation coefficient and $\mathrm{p}$ value was calculated to check the correlations between the degree of improvement in trainees' self-assessment data from baseline and the degree of improvement in physicians' assessment data from baseline among regular and intervention groups.

\section{RESULTS}

\section{Basic characteristics of medical clerks}

There was no difference among the regular, intervention and control groups in regard to the mean age, distribution of gender, percentage of trainees receiving training in communication skills within 6 months before participating the study, percentage of trainees receiving training of preprocedural communication skills within 6 months before participating in the study, percentage of trainees with experience communicating with patients' family regarding the details of medical procedures including central venous catheterisation, lumbar puncture, bronchoscopy, thoracentesis and tracheostomy (table 3).

\section{The OSCAR preprocedural communication framework-based} intervention module was generally well accepted by trainees There was no difference in the baseline degree of the medical clerks' agreement with skills-related statements 
Table 2 Baseline, end-of-rotation and follow-up assessment form for medical clerks and physicians

The first part is skills-related You are (trainee is) familiar with skills related to providing information (preparation, periprocedural statements 1-3 cooperation and postprocedural precaution) regarding medical procedures to patients You have (trainee has) confidence in providing information (preparation, periprocedural cooperation and postprocedural precaution) regarding medical procedures to patients You are (trainee is) not anxious about providing information (preparation, periprocedural cooperation and postprocedural precaution) regarding medical procedures to patients

The second part is framework-related statements 1-3
You are (trainee is) familiar with the regular or OSCAR framework, you have (trainee has) confidence in using the regular or OSCAR framework

You have (trainee has) confidence in using the regular or OSCAR framework

You are (trainee is) not anxious about using the regular or OSCAR framework

The third part is the competency to use steps in framework-related statements 1-5 (*'regular' and 'control' groups answer the statement 1-1, 2-1, 3-1, 4-1, 5-1 and the 'intervention' group answer the statement 1-2, 2-2, 3-2, 4-2, 5-2) OSCAR framework OSCAR framework" subjects'
Statement 1-1: you (trainee) can use the 'step-1: setting the stage' of the regular framework'

Statement 1-2: you (trainee) can use the 'O-step: opening with what, when, why and how' of the

Statement 2-1: you (trainee) can use the 'step-2: eliciting information' of the regular framework Statement 2-2: you (trainee) can use the 'S-step: explaining Subject to be communicated' of the

Statement 3-1: 'you (trainee) can use the 'step-3:giving information' of the regular framework' Statement 3-2: 'you (trainee) can use the 'C-step:Check patients understanding about the

Statement 4-1: 'you (trainee) can use the 'step-4.understanding the patient's perspective' of the regular framework'

Statement 4-2: 'you (trainee) can use the 'A-step: Aid patient understanding with audiovisual materials'

Statement 5-1: 'you (trainee) can use the 'step-5:ending the encounter' of the regular framework' Statement 5-2: 'you (trainee) can use the 'R-step:Recheck patient understanding' of the OSCAR framework'

Responders answer each statement with 5-point Likert scale (5=strongly agree, $4=$ agree, $3=$ neutral, $2=$ did not agree,

$1=$ strongly disagree)

Table 3 Basal characteristics of three groups
Control group medical clerks $(n=48)$

clerks $(n=48) \quad$ medical clerks $(n=48)$

\begin{tabular}{llll}
\hline Age (years) & $21.5 \pm 1.2$ & $22.8 \pm 0.9$ & $21.9 \pm 1.4$ \\
Gender (female/male) & $21 / 27(44 / 56 \%)$ & $23 / 25(48 / 52 \%)$ & $16 / 32(33 / 67 \%)$ \\
$\%$ of trainees receiving training of & $46(96 \%)$ & $47(97 \%)$ & $44(92 \%)$
\end{tabular}

communication skills within 6 months before

participating the study

$\%$ of trainees receiving training of preprocedural $4 / 48(8 \%)$

communication skills within 6 months before

participating the study

$\%$ of trainees who have experience

$12 / 48(25 \%)$

$5 / 48(10 \%)$

$3 / 48(6 \%)$

communicating with patients about the details

of any one of the medical procedures including

either central venous catheterisation, lumbar

puncture, bronchoscopy, thoracentesis

or tracheostomy within 6 months before

participating the study among the three groups (figure 2A). In comparison to the baseline stage, the degree of agreement of regular and intervention group' trainees to the aforementioned skills-related statements significantly increased at end-ofrotation stage. The degree of improvement in trainees' agreement with the aforementioned skills-related statements 1-2 was greater in the intervention group than that in the regular group (regular vs intervention group: skill-1 statement: $48 \% \pm 3 \%$ vs $105 \pm 9 \%, \mathrm{p}<0.001)$; skill-2 statement: $50 \% \pm 9 \%$ vs $71 \pm 11 \%, \mathrm{p}<0.05$, figure $2 \mathrm{~A})$.

There were no differences among the three group in the degree of the medical clerks' baseline agreement with 
Trainees' self-assessments
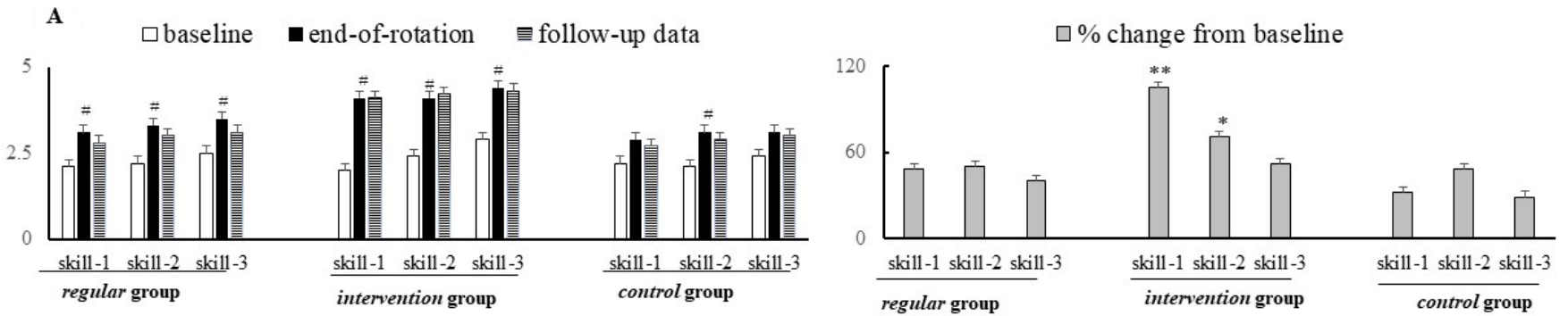

....familiar with [skills-related statement-1 (skill 1)], have confidence in (skill 2), not anxious about (skill 3) giving information (preparation, peri-procedural cooperation, and post-procedural precaution) about medical procedures to patients

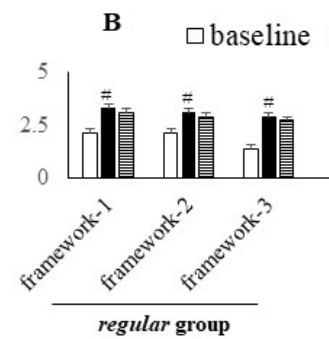

regular group
- end-of-rotation 目 follow-up data

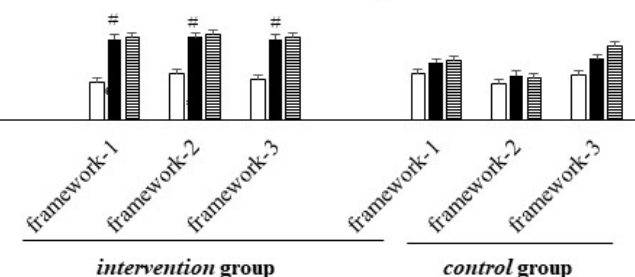

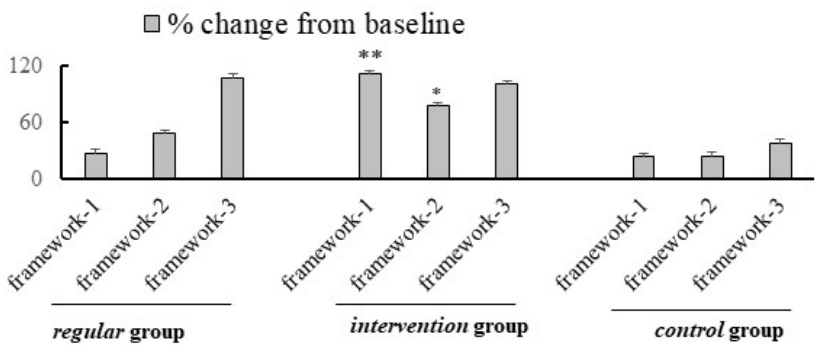

familiar with using (framework-related statement-1: framework 1), have confidence in using (framework 2), not anxious about using (framework 3) framework Figure 2 Comparison of the baseline (introductory class) and end-of rotation (end-of rotation class) degree of medical clerks' agreement with the competency-related statements. (A) Trainees' agreement with the skills-related statements for preprocedural communication competency; (B) trainees' agreement with the framework-related statements for preprocedural communication competency. ${ }^{*} \mathrm{P}<0.05$ or ${ }^{* \star} \mathrm{p}<0.001$ vs regular group; $\# \mathrm{p}<0.05$ vs baseline data; STAT, statement.

the framework-related statements (figure 2B). In comparison with the baseline data, the degree of the regular and intervention group trainees' end-of-rotation agreement with the aforementioned framework-related statements significantly increased. The degree of improvement in trainees' agreement with the framework-related statement 1-2 was greater in the intervention group than that in the regular group ((regular vs intervention group: framework-1 statement: $27 \% \pm 5 \%$ vs $111 \pm 13 \%, \mathrm{p}<0.001)$; framework-2 statement: $48 \% \pm 2 \%$ vs $77 \pm 9 \%, \mathrm{p}<0.05$, figure 2B). The trainees' end-of-rotation agreement with the skills and framework-related statements 1 and 3 did not differ from the baseline in the control group.

\section{Each step in the OSCAR framework is well accepted by the intervention group trainees}

Figure 3A-B reveals that the baseline trainees' agreement with the statements regarding their competency to use each step of either the regular or OSCAR framework did not differ among the three groups. Particularly, the intervention group's baseline competency to use the "Astep" of the OSCAR framework is relatively lower but not reach the statistical significance than for other steps of the OSCAR framework (figure 3B). At the end-of-rotation stage, regular and intervention group trainees' agreement with the statements that they could use each step of the regular or the OSCAR framework were increased. At the end-of-rotation stage, the ability of the intervention group to use the 'A-step' of the OSCAR framework was significantly improved $(229 \% \pm 13 \%$ improved from baseline, $\mathrm{p}<0.001$ compared with the degree of improvement in other steps, figure 3B). The control group trainee's agreement with the statement regarding their competency of using each step of the regular frameworks did not differ between the baseline and end-of-rotation stages.

\section{Physicians reported that the acceptability of trainees to} framework-based communication is higher in the intervention group than the regular group

Similar to the changing trends of the trainees' competency by physician assessments were similar to the data of trainees' self-assessment (figure 2A). The baseline trainees' competency (familiarity, confidence, and anxiety) of giving preprocedural communication, using the regular or OSCAR framework, and using each step of the regular or OSCAR framework were similar between the regular and intervention groups (figures $4 \mathrm{~A}-\mathrm{B}$ and $5 \mathrm{~A})$. Significantly, the degree of improvement from baseline was greater in the intervention group than those in the regular group (regular vs intervention group: skill-1 statements: $21 \% \pm 7 \%$ vs $100 \% \pm 9 \%, \mathrm{p}<0.001)$; framework-1 statements: $45 \% \pm 3 \%$ vs $100 \% \pm 11 \%, \mathrm{p}<0.001$, figure $4 \mathrm{~A}-\mathrm{B})$. In the intervention group, the physiciansassessed end-of-rotation data revealed that the improvement was highest in the statement of "trainee can use the 'A-step: Aid patient understanding with audiovisual materials' compared with the other four 'O-step, S-step, C-step, 
Trainees' self-assessments

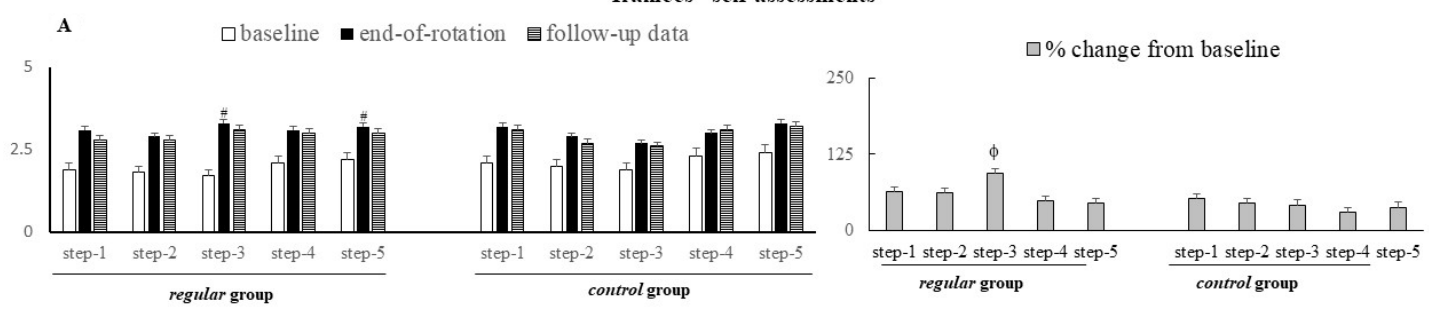

can use the "step-1.setting the stage", the "step-2.eliciting information", the "step-3.providing information", "step-4.understanding the patient's perspective", "step-5.ending the encounter" of the regular framework (table 1)
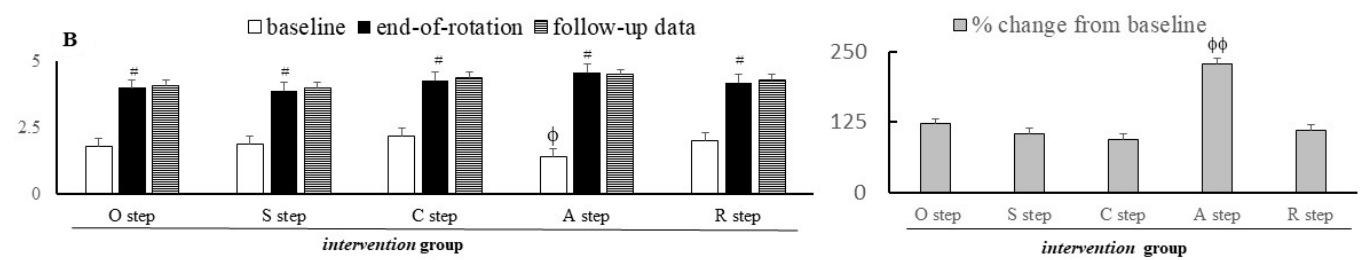

can use the "O-step: opening with what, when, why and how", "S-step: clarifying the subject to be discussed", "C-step: check for patients understanding of the subjects", "A-step: Aid patient understanding with audiovisual materials", "ㅈ-step: recheck the patient's understanding" of OSCAR framework in intervention module

Figure 3 Comparison of the baseline and end-of rotation degree of medical clerks' agreement with the self-assessed competency of using each step of the framework. (A) Regular and control group trainees' agreement with the statements that they can use each step of the regular framework; (B) Intervention group trainees' agreement with the statements that they can use each step of the OSCAR framework. \#p<0.05 vs baseline data; STAT, statement.

Assessment of trainees by physicians
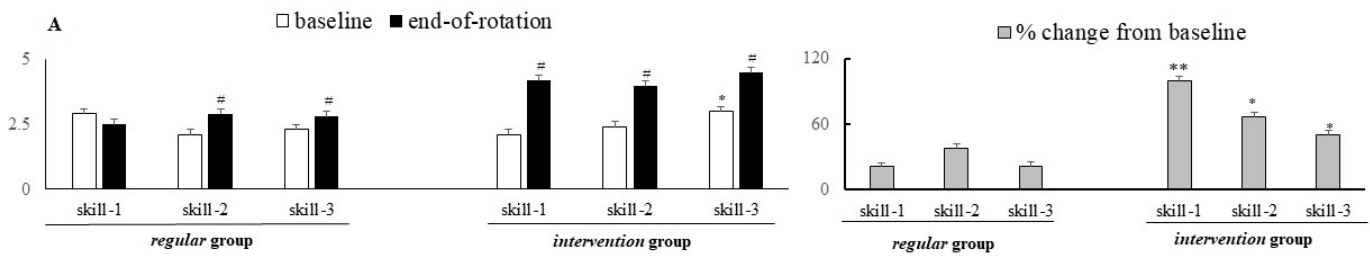

....familiar with [stat.1: statement-1], have confidence in (statement-2), not anxious about (statement-3) giving information (preparation, periprocedural cooperation, and post-procedural precaution) about medical procedures to patients
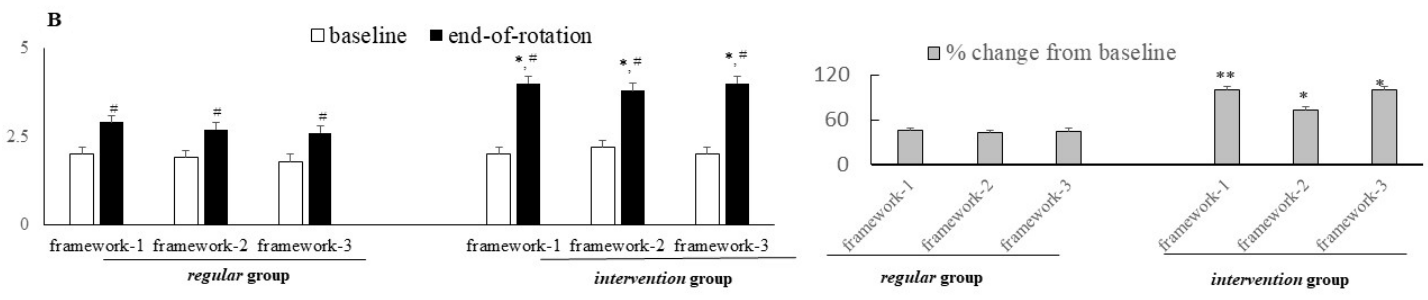

familiar with using (statement-1), have confidence in using (statement-2), not anxious about using (statement-3) communication framework

Figure 4 Comparison of the baseline and end-of rotation degree of physicians' agreement with the trainees' competencyrelated statements. (A) Physicians' agreement with the trainees' skills-related statements for preprocedural communication competency; (B) physicians' agreement with the trainees' framework-related statements for preprocedural communication competency. ${ }^{*} \mathrm{P}<0.05$ or ${ }^{* *} \mathrm{p}<0.001$ vs regular group; $\# \mathrm{p}<0.05$ vs baseline data.

R-step' statements (A-step: 170\% vs O-step: 129\%, S-step: 111\%; C-step:91\% ; R-step: 100\%).

The trainees self-assessed degree of improvement from baseline at end-of-rotation stage in the intervention group was correlated with physician assessment

At the end-of-rotation stage, the degree of improvement in regular group trainees' self-assessment data from baseline was higher than the degree of improvement in physicians' assessment data from baseline (figure 5B). Notably, the degree of improvement in the self-assessment data of the intervention group trainees' from baseline was similar to the degree of improvement in physicians' assessment data from baseline. Further, correlation analysis revealed that, in the intervention group, the degree of improvement of the end-of-rotation data of trainees' self-assessment from baseline was significantly correlated with the degree of the improvement in physicians' assessment data in the aspects of skills, framework, and steps in 
A

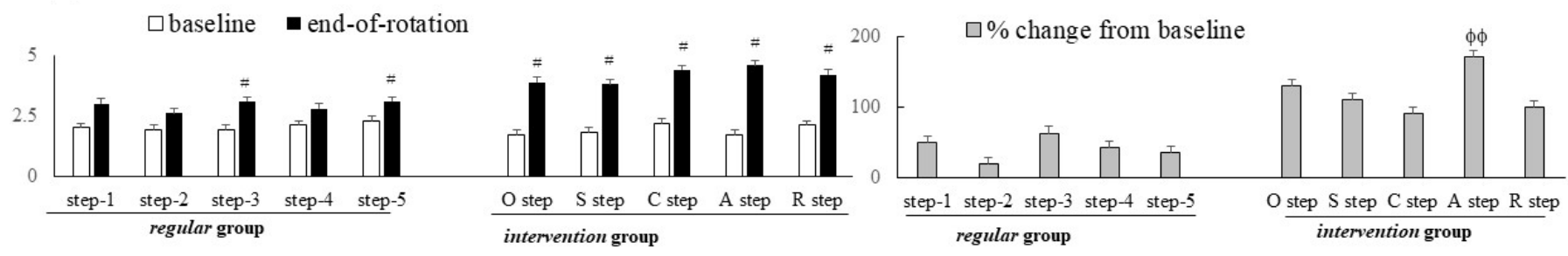

.can use the "step-1.setting the stage", the "step-2.eliciting information", the "step-3.providing information", "step-4.understanding the patient's perspective", "step-5.ending the encounter" of the regular framework

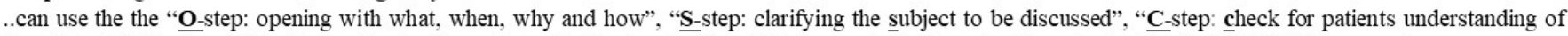
the subjects", "ㅅ-step: Aid patient understanding with audiovisual materials", "ㅁ-step: recheck the patient's understanding" of OSCAR framework

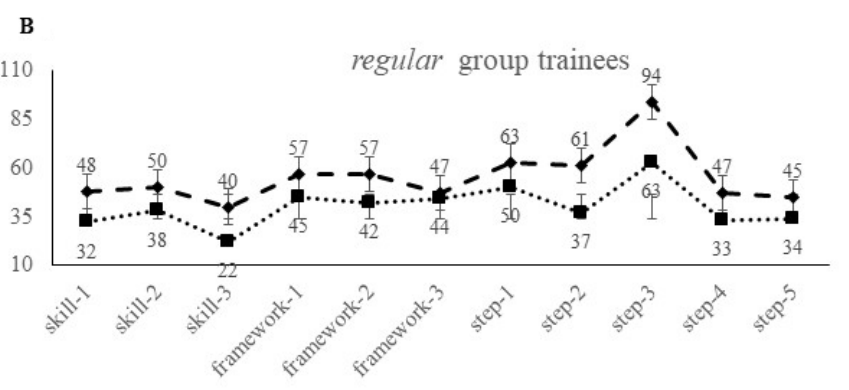

- - mean $\%$ change of self-assessed end-of-rotation competency from baseline

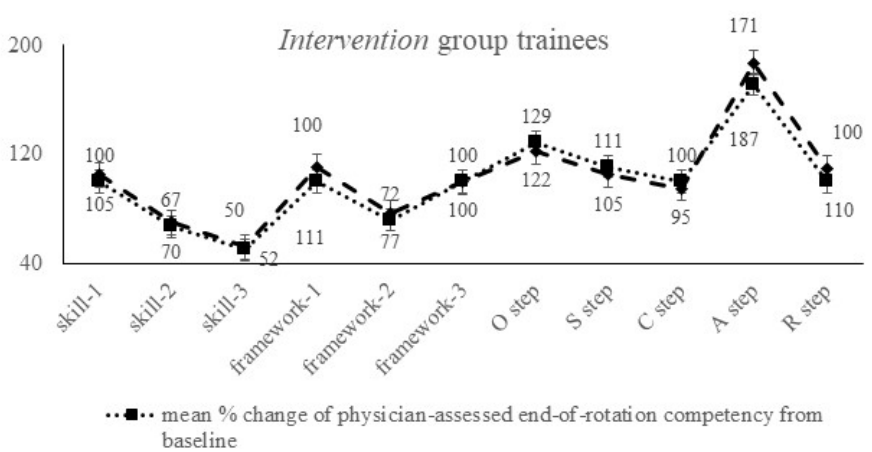

baseline

Figure 5 Per cent change of end-of rotation data from baseline data for trainees' self-assessed competency and corresponding physicians-assessed trainees' competency. (A) Degree of physicians' agreement to the statement regarding trainees' competency to use each step of regular or OSCAR framework; (B) comparison of the percent changes of end-ofrotation data from baseline data for trainees' self-assessed competency and corresponding physicians-assessed trainees' competency. $\varnothing \varnothing: \mathrm{p}<0.001 \mathrm{vs}$. other groups, $\# \mathrm{p}<0.05 \mathrm{vs}$ baseline data.

framework ( $\mathrm{R}=0.872, \mathrm{p}<0.01 ; \mathrm{R}=0.813, \mathrm{p}<0.001 ; \mathrm{R}=0.914$, $\mathrm{p}<0.001$, figure 6$)$. Nonetheless, in the regular group, the degree of improvement of the end-of-rotation data from baseline of trainees' self-assessment was not significantly correlated with the degree of the improvement in physicians' assessment data in the aspects of skills, framework and steps in framework (figure 6).

OSCAR framework is well accepted by trainees and motivates them to integrate the acquired preprocedural communication skills into primary care

Overall, the email response rate to the follow-up assessment 4 weeks after training class was $85 \%, 87 \%$ and $93 \%$ among the regular, control and intervention groups, respectively. The follow-up data revealed that a higher percentage $(85 \%)$ of the intervention group trainees had applied the acquired skills in primary care than trainees in the regular (65\%) and control $(40 \%)$ group (table 4). Notably, in comparison to the data of the endof-rotation stage, the intervention group's agreement with the skill-related and framework-related statements did not decrease at the follow-up stage. Nonetheless, the regular group' agreement to the above skill-related and framework-related statements were decreased at follow-up stage (figure 2A-B). These results indicated that the OSCAR framework-based intervention module had good retention training effects among the intervention group' trainees. Moreover, the degree of intervention group' agreement to the statement of 'your patients are satisfied with your preprocedural communication skills' is higher than that of regular and control groups (table 4).

\section{DISCUSSION}

In our study, both in the regular framework-based and OSCAR framework-based intervention modules, the teaching schedule included didactic approach and small group role-play plus discussion. The didactic approach has the advantages of (1) it efficiently presents core concepts for large numbers of learners; (2) it requires minimal time and faculty resources to deliver content to a wide range of learners and (3) it provides an opportunity for learners to efficiently use demonstrated skills. Small group role-play plus discussion, on the other hand, has the following advantages: (1) it presents multiple scenarios showing a range of approaches and patient responses; (2) trainees receive skills practice with feedback from faculty and peers and (3) it provides learners with the opportunity to discuss issues, skills and concerns. ${ }^{22-24}$

SPs, who are actors trained to reliably and reproducibly enact a patient scenario, allowing students to practice this challenging communication task without the concern of causing harm to an actual patient. ${ }^{24} \mathrm{SP}$ simulations are effective in teaching and assessing communication skills. ${ }^{25}$ In both modules of this study, the SPs had been involved in the demonstration and role play on the introductory 


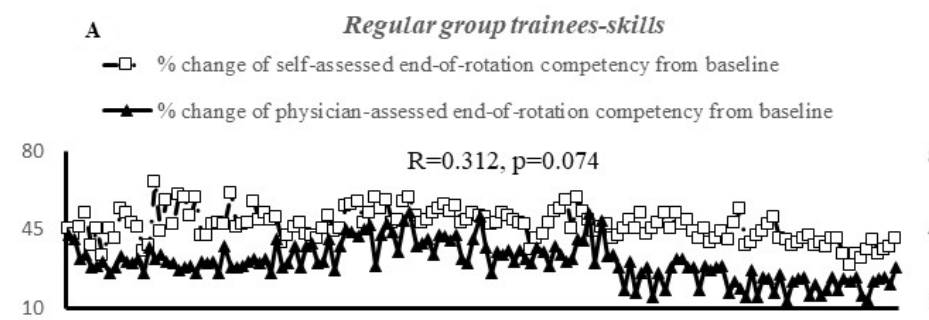

B

Regular group trainees-framework

-. . $\%$ change of self-assessed end-of-rotation competency from baseline

$\longrightarrow \%$ change of physician-as sessed end-of-rotation competency from baseline $\mathrm{R}=0.155, \mathrm{p}=0.068$

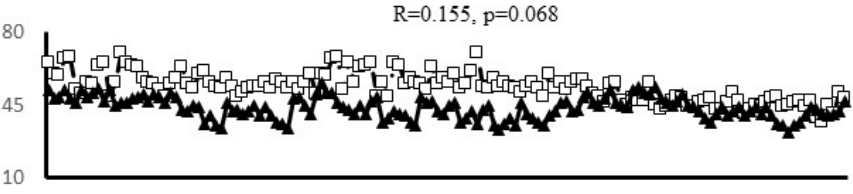

Regular group trainees-steps of framework

- $\cdot \%$ change of self-assessed end-of-rotation competency from baseline

C $\longrightarrow$ \% change of physician-assessedend-of-rotation competency from baseline

D - $\square \cdot \%$ change of self-assessed end-of-rotation competency from baseline
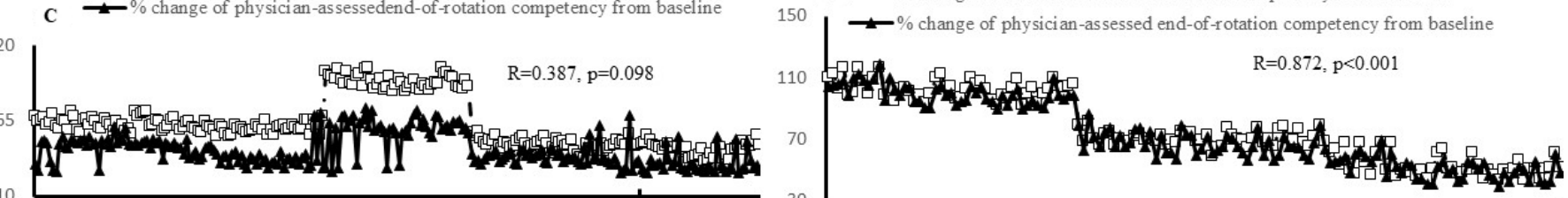

Intervention group trainees-framework

E - $\quad$ - \% change of self-assessed end-of-rotation competency from baseline

Intervention group trainees-steps in framework

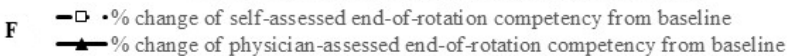

$\longrightarrow \%$ change of physician-assessedend-of-rotation competency from baseline
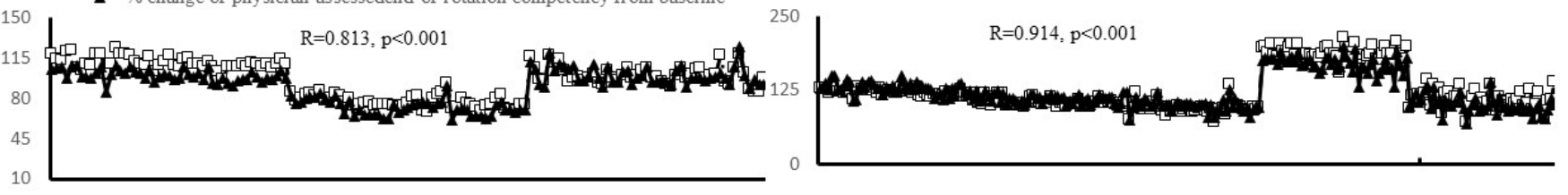

Figure 6 Correlation between the per cent change of different aspects of the end-of rotation data from baseline data of trainees' self-assessed competency and corresponding physicians-assessed trainees' competency. (A) The skills aspect in the regular group; (B) the framework aspect in the regular group; (C) the steps of framework aspect in the regular group; (D) the skills aspect in the intervention group; (E) the framework aspect in the intervention group; (F) the steps of framework aspect in the intervention group.

Table 4 Follow-Up (4 weeks after end-of-rotation class) competency of three groups

Regular group medical clerks $(\mathbf{n}=48)$
Intervention group medical clerks $(n=48)$

$\%$ of trainees reported that they had applied the $31 / 48$ (65\%)

$41 / 48(85 \%)$

Control group medical clerks $(n=48)$

acquired skills in primary care

Average degree of trainees' agreement with the $2.9 \pm 0.6$

$4.4 \pm 0.5^{*}$

$19 / 48(40 \%)$

statement 'your patients are satisfied with your

preprocedural communication skills'

5-point likert scale from 1 to 5 ( $\square$ very agree (5), $\square$ agree (4), $\square$ neutral (3), $\square$ not agree (2), $\square$ not very agree (1).

${ }^{*} \mathrm{P}<0.05$ vs regular group.

and end-of-rotation classes. Through role play with SPs, physicians can directly assess the improvement in trainees' competency in preprocedural communication skills. In the control group, physicians assessed trainees' baseline and end-of-rotation competency via observation in clinical wards.

In our study, both regular and OSCAR frameworkbased small-group training is well accepted by trainees and increased their confidence in providing preprocedural communication. Moreover, the regular and intervention group' trainees reported that additional small-group training modules were useful in increasing their confidence in handling difficult communication. Most trainees desire more opportunities to role-play communication skills with SPs.
Self-assessment plays an important role in supporting the development of reflection and self-awareness skills. ${ }^{26}$ Therefore, researchers have suggested that the data from medical clerks' self-assessment is a record of the process of self-reflection. For medial trainees, self-assessment is suggested as a way of identifying one's strengths and weaknesses to guide goal setting and enhance self-efficacy. ${ }^{26}{ }^{27} \mathrm{It}$ is believed that students who maintain a more active role in the learning process are able to assess their own performance more objectively. ${ }^{28}$ So, in our study, both the regular and OSCAR framework-based modules increased medical clerks' self-assessed competency for providing preprocedural communication after training.

It is indicated that the direct involvement of learners in assessing their work is highly effective in enhancing 
learning. Familiarity with an activity has been shown to positively affect trainees' ability to self-assess of the performance of their task. ${ }^{29}$ Teachers' evaluation brings more solid results. ${ }^{30}$ In our study, most of the medical clerks reported that they need to assess their readiness about audiovisual materials that incorporated in OSCAR framework before using it. Probably, this involvement increases their familiarities with skills, framework and steps in OSCAR communication framework. Notably, in intervention group, the degree of the end-of-rotation data of trainees self-assessed improvement from baseline was correlated with the degree of improvement the data of physicians' assessment.

Studies show that patients often feel that they do not receive satisfactory information from their healthcare provider. ${ }^{31}$ Increasing patients' families understanding of medical information through effective communication can enhance patient satisfaction and outcomes. ${ }^{31}{ }^{32}$ In particular, audiovisual materials have been suggested to increase the effectiveness of preprocedural communication with patients with severe illness. ${ }^{33}$

In medical communication, specific information needs can vary individually among patients. ${ }^{32-35}$ Thus, it is important for healthcare providers to assess patients' needs before providing medical information in patient-centred communication. ${ }^{34-36}$ Therefore, in our newly designed OSCAR framework, the process of communication is emphasised.

This prospective study was limited to a single site with relatively small sample size to generalise. Moreover, this study was limited by the lack of evaluation of the degree of the transfer of medical clerks' acquired skill to clinical practice by direct work-place assessments of patient satisfaction. In order to avoid 'self-fulfilling prophecy' or bias caused, the effectiveness of the intervention should be addressed on patients' level such as whether patients less anxious after the preprocedural information brought by the trainee. So, the methodological limitations of the study are that the competency of medical clerks' skills in giving preprocedural communication was observed directly by physicians in class rather than in clinical ward. To monitor the transfer of medical students' acquired skills to primary care, workplace assessments and surveys of patients' satisfaction are necessary in the future. Alternatively, the effects on patient level can be evaluated by qualitative assessment by teachers whose are blinded for the intervention rather than students' self-assessment in our study.

\section{CONCLUSIONS}

Overall, this study reported the acceptability of additional training of medical clerks for using a preprocedural communication framework and material in primary care. The OSCAR framework-based intervention module, which emphasises the use of audiovisual materials, is well accepted by medical clerks. The follow-up email was sent to medical students, and the intervention group' medical clerks reported that they continuously applied the acquired preprocedural communication skills and framework in clinical practice. Overall, the trainees reported that their primary care patients were satisfied with their preprocedural communication skills.

\section{Author affiliations}

${ }^{1}$ Department of Critical Care Medicine, Taipei Veterans General Hospital, Taipei, Taiwan

${ }^{2}$ Medical college, National Yang Ming Chiao Tung University, Taipei, Taiwan ${ }^{3}$ Faculty of Medicine, National Yang Ming Chiao Tung University, Taipei, Taiwan ${ }^{4}$ Department of Emergency, Taipei Veterans General Hospital, Taipei, Taiwan ${ }^{5}$ Department of Medical Education, Taipei Veterans General Hospital, Taipei, Taiwan ${ }^{6}$ Medical Innovation and Research Center, Clinical Innovation Center, Taipei Veterans General Hospital, Taipei, Taiwan

${ }^{7}$ Clinical Skill Training Center, Taipei Veterans General Hospital, Taipei, Taiwan ${ }^{8}$ Department of Internal Medicine, Shin Kong Wu Ho Su Memorial Hospital, Taipei, Taiwan

${ }^{9}$ Medical education, Taipei Veterans General Hospital, Taipei, Taiwan

${ }^{10}$ Division of Gastroenterology and Hepatology, Department of Medicine, Taipei Veterans General Hospital, Taipei, Taiwan

${ }^{11}$ Division of metabolism and endoscrinology, Department of Medicine, Taipei Veterans General Hospital, Taipei, Taiwan

Acknowledgements The authors express their gratitude to all trainees and faculties for their input for this article.

Contributors All authors actively participated in analysis of the research results. Y-YY, D-HC, C-TC and T-HL were responsible for study design. Y-YY, D-HC, T-YW, $\mathrm{C}-\mathrm{CH}$ and $\mathrm{T}-\mathrm{HL}$ participated in the design and data analysis of questionnaires. $\mathrm{Y}-\mathrm{YY}$, D-HC, C-TC, T-YW, T-HL, S-SH, S-YK, M-CH, WH-H participated in the creation and management of the database. Y-YY, S-YK, C-HC, M-CH and WH-H were responsible for the statistical analysis and writing of the manuscript. $Y-Y Y$ and $D-H C$ are guarantor of this article.

Funding This work was supported by the Taipei Veteran General Hospital, Grant. 110EA-007, 111EA-009, V111C-018, by the Ministry of Education with grant №. PMN1100719, by the Ministry of Science and Technology with the grant No. MOST 109-2314-B-010-032-MY3 and grant No. MOST-110-2511-H-A49A-504 -MY3.

\section{Competing interests None declared.}

Patient and public involvement Patients and/or the public were not involved in the design, or conduct, or reporting, or dissemination plans of this research.

Patient consent for publication Consent obtained directly from patient(s)

Ethics approval Ethical approval (IRB No. 2018-07-030AC and 2019-12-007ACF) was granted by the ethics committee of the Taipei Veteran General Hospital. All participants were informed about the importance and advantage of this intervention for their competencies and informed consent was obtained from all participants and data were collected. The study complied with the Declaration of Helsinki.

Provenance and peer review Not commissioned; externally peer reviewed.

Data availability statement All data relevant to the study are included in the article or uploaded as online supplemental information.

Open access This is an open access article distributed in accordance with the Creative Commons Attribution Non Commercial (CC BY-NC 4.0) license, which permits others to distribute, remix, adapt, build upon this work non-commercially, and license their derivative works on different terms, provided the original work is properly cited, appropriate credit is given, any changes made indicated, and the use is non-commercial. See: http://creativecommons.org/licenses/by-nc/4.0/.

\section{ORCID iD}

Ying-Ying Yang http://orcid.org/0000-0002-7719-0397

\section{REFERENCES}

1 Schöpf AC, Vach W, Jakob M, et al. Routine patient surveys: patients' preferences and information gained by healthcare providers. PLoS One 2019;14:e0220495.

2 Lim L, Chow P, Wong C-Y, et al. Doctor-Patient communication, knowledge, and question prompt Lists in reducing preoperative anxiety: a randomized control study. Asian J Surg 2011;34:175-80. 
3 Goldberger JJ, Kruse J, Kadish AH, et al. Effect of informed consent format on patient anxiety, knowledge, and satisfaction. Am Heart J 2011;162:780-5.

$4 \mathrm{Kim} \mathrm{B}-\mathrm{H}$, Kang $\mathrm{H}-\mathrm{Y}$, Choi E-Y. Effects of handholding and providing information on anxiety in patients undergoing percutaneous vertebroplasty. J Clin Nurs 2015;24:3459-68.

5 Mendoza BA, Fortier MA, Trinh LN, et al. Factors impacting parental and child satisfaction in the perioperative setting. Paediatr Anaesth 2021;31:932-43.

6 Roter DL, Hall JA, Kern DE, et al. Improving physicians' interviewing skills and reducing patients' emotional distress. A randomized clinical trial. Arch Intern Med 1995;155:1877-84.

7 Swing SR. The ACGME outcome project: retrospective and prospective. Med Teach 2007;29:648-54.

8 Hawkins RE, Lipner RS, Ham HP, et al. American Board of medical specialties maintenance of certification: theory and evidence regarding the current framework. J Contin Educ Health Prof 2013;33 Suppl 1:S7-19.

9 Seale J, Ragbourne SC, Purkiss Bejarano N, et al. Training final year medical students in telephone communication and prioritization skills: an evaluation in the simulated environment. Med Teach 2019;41:1023-8.

10 Lörwald AC, Lahner F-M, Nouns ZM, et al. The educational impact of Mini-Clinical evaluation exercise (Mini-CEX) and direct observation of procedural skills (DOPS) and its association with implementation: a systematic review and meta-analysis. PLoS One 2018;13:e0198009.

11 Lörwald AC, Lahner F-M, Mooser B, et al. Influences on the implementation of Mini-CEX and DOPS for postgraduate medical trainees' learning: a grounded theory study. Med Teach 2019;41:448-56.

12 Haidet P, Dains JE, Paterniti DA, et al. Medical student attitudes toward the doctor-patient relationship. Med Educ 2002;36:568-74.

13 Cinar O, Ak M, Sutcigil L, et al. Communication skills training for emergency medicine residents. Eur J Emerg Med 2012;19:9-13.

14 Skillings JL, Porcerelli JH, Markova T. Contextualizing SEGUE: evaluating residents' communication skills within the framework of a structured medical interview. J Grad Med Educ 2010;2:102-7.

15 Accreditation Council for Graduate Medical Education (ACGME) and American Board of Medical Specialties. Toolbox of assessment methods, version 1.1, 2000. Available: http://www.acgme.org/ Outcome/assess/ToolTable.pdf [Accessed 08 Jul 2009].

16 Epp DA, Kubota T, Yoshida M, et al. Promoting patient care through communication training in a pre-clerkship pharmacy education course in Japan. Am J Pharm Educ 2019;83:6745.

17 Vijn TW, Fluit CRMG, Kremer JAM, et al. Involving medical students in providing patient education for real patients: a scoping review. $J$ Gen Intern Med 2017;32:1031-43.

18 Wilson NA, Reich AJ, Graham J, et al. Patient perspectives on the need for implanted device information: implications for a post-procedural communication framework. Health Expect 2021;24:1391-402

19 Herrera-Espiñeira C, Rodríguez del Aguila MdelM, Rodríguez del Castillo M, et al. Relationship between anxiety level of patients and their satisfaction with different aspects of healthcare. Health Policy 2009;89:37-45.

20 Snyder-Ramos SA, Seintsch H, Böttiger BW, et al. Patient satisfaction and information gain after the preanesthetic visit: a comparison of face-to-face interview, brochure, and video. Anesth Analg 2005;100:1753-8.

21 Salzwedel C, Petersen C, Blanc I, et al. The effect of detailed, video-assisted anesthesia risk education on patient anxiety and the duration of the preanesthetic interview: a randomized controlled trial. Anesth Analg 2008;106:202-9.

22 Girvan C, Conneely C, Tangney B. Extending experiential learning in teacher professional development. Teach Teach Educ 2016;58:129-39.

23 Villamizar AG, Mejía G. Fostering learner autonomy and critical reflection through digital video-journals in a university foreign language course. Reflective Practice 2019;20:187-200.

24 Geoffroy PA, Delyon J, Strullu M, et al. Standardized patients or conventional Lecture for teaching communication skills to undergraduate medical students: a randomized controlled study. Psychiatry Investig 2020;17:299-305.

25 Kaufman DM, Laidlaw TA, Langille D, et al. Differences in medica students' attitudes and self-efficacy regarding patient-doctor communication. Acad Med 2001;76:188.

26 Papinczak T, Young L, Groves M, et al. An analysis of peer, self, and tutor assessment in problem-based learning tutorials. Med Teach 2007;29:e122-32.

27 Eva KW, Regehr G. Self-Assessment in the health professions: a reformulation and research agenda. Acad Med 2005;80:S46-54.

28 Milles LS, Hitzblech T, Drees S, et al. Student engagement in medical education: a mixed-method study on medical students as module co-directors in curriculum development. Med Teach 2019;41:1143-50.

29 Mort JR, Hansen DJ. First-year Pharmacy Students' SelfAssessment of Communication Skills and the Impact of Video Review. Am J Pharm Educ 2010;74:78.

30 Papinczak T, Young L, Groves M, et al. An analysis of peer, self, and tutor assessment in problem-based learning tutorials. Med Teach 2007;29:e122-32.

31 Timmins F. Exploring the concept of 'information need'. Int J Nurs Pract 2006;12:375-81.

32 Behar-Horenstein LS, Guin P, Gamble K, et al. Improving patient care through patient-family education programs. Hosp Top 2005;83:21-7.

33 Carey M, Schofield P, Jefford M, et al. The development of audiovisual materials to prepare patients for medical procedures: an oncology application. Eur J Cancer Care 2007;16:417-23.

34 Johnson M, Tod AM, Brummell S, et al. Prognostic communication in cancer: a critical interpretive synthesis of the literature. Eur $\mathrm{J}$ Oncol Nurs 2015;19:554-67.

35 King A, Hoppe RB. "Best practice" for patient-centered communication: a narrative review. J Grad Med Educ 2013;5:385-93.

36 Madula P, Kalembo FW, Yu H, et al. Healthcare provider-patient communication: a qualitative study of women's perceptions during childbirth. Reprod Health 2018;15:135. 